
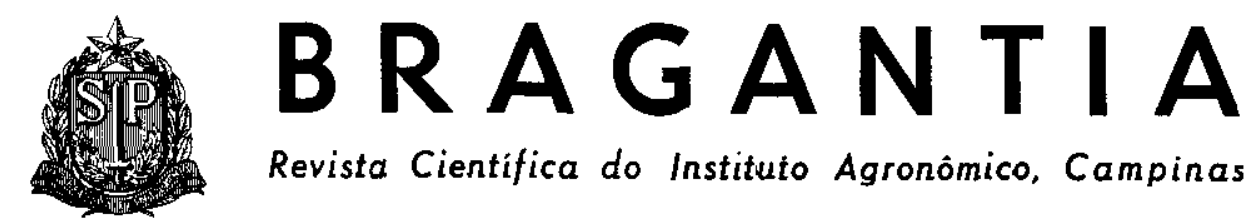

Revista Científica do Instituto Agronômico, Campinas

Vol. 41

Campinas, abril de 1982

Artigo n. ${ }^{\circ} 8$

\title{
ESTIMATIVA DA CAPACIDADE PRODUTIVA DE NOVAS LINHAGENS DE AMENDOIM (')
}

\author{
Antonio Sidney Pompeu, Seção de Genética, Instituto Agronômico
}

\section{RESUMO}

A produtividade de novas linhagens de amendoim (Arachis hypogaea) foi avaliada em 36 experimentos, tendo como controle o cultivar Tatu, no cultivo das águas, em 1977, 1978 e 1979, no Centro Experimental de Campinas. Essas linhagens, inicialmente em número de $323 \mathrm{em} 1977$ e 1978, das quais apenas 107 foram incluidas nos ensaios de 1979, são originárias de plantas individuais selecionadas nos cruzamentos Tatuí $x$ Roxo 80-1, Tatu x Introđuçōes, Tatu x Tatú, Tatu x Tatu-Amarelo, Tatu x Roxo 47, Tatu $x$ Roxo 80-1, Tatu $x$ (Tatuí $x$ Arachis monticola), Tatu $x$ (Tatui $x$ A. villosa) e (Tatu $\mathrm{x}$ Roxo 80-1) x (Tatuí x A. monticola). As anólises estatísticas dos dados obtidos para as linhagens comuns aos ensaios de 1977, 1978 e 1979 e 1978 e 1979 , mostraram que 22 delas, com produçōes médias de 4.219 a $6.445 \mathrm{~kg} / \mathrm{ha}$, foram superiores às observadas para o cultivar Tatu, as quais variaram de $3.456 \mathrm{a} 4.887 \mathrm{~kg} / \mathrm{ha}$. Os efeitos de ano e da interaçāo tratamento $\mathrm{x}$ ano foram significativos em onze $\mathrm{e}$ três das treze análises conjuntas, respectivamente. As melhores linhagens serão incluídas nos ensaios regionais a serem instalados nas principais regióes produtoras paulistas, enquanto algumas teräo suas sementes aumentadas em quantidade e colocadas à disposiçāo dos agricultores.

\section{INTRODUÇÃO}

A produção brasileira de amendoim em 1978 foi 325.000 toneladas, obtidas em uma área de $252.000 \mathrm{ha}$, com um rendimento médio de $1.293 \mathrm{~kg} / \mathrm{ha}$ (1). Dos Estados brasileiros, destacaram-se São Paulo, Paraná e Mato Grosso, que produziram, em $1978,227.400,50.406$ e 24.948 toneladas, em $172.400,40.266 \mathrm{e}$ 21.106 ha respectivamente. Os ren- dimentos observados, 1.319, 1.252 e $1.182 \mathrm{~kg} /$ ha (2), são considerados baixos quando comparados com 2.754 e $2.958 \mathrm{~kg} / \mathrm{ha}$ obtidos nos Estados Unidos em 1977 e 1978 (1), embora o nível de tecnologia usado seja diferente. Entre os fatores responsáveis pelo baixo rendimento, destacam-se o preparo inadequado do solo, o baixo uso de adubos e corretivos e o controle ineficiente de agentes patogênicos e pragas. 
Embora o cultivar Tatu, praticamente o único plantado em grande escala no Estado de São Paulo, apresente boa capacidade produtiva, procura-se, no programa de melhoramento em andamento na Seção de Genética, desenvolver novos cultivares que o superem em produtividade. visando aumentar o rendimento desta leguminosa em nossas condições. Assim, várias linhagens foram obtidas a partir de cruzamentos, sendo suas produções, comparadas com a do 'Tatu', mostradas neste trabalho.

\section{MATERIAL E METODO}

As linhagens de amendoim estudadas em ensaios instalados no cultivo das águas de 1977, 1978 e 1979, no Centro Experimental de Campinas, são resultantes de seleçōes individuais efetuadas nos seguintes cruzamentos: 'Tatuí' x Roxo 80-1, Tatu (linhagem 34) $x$ Introduções $(357 \mathrm{G}, 525 \mathrm{G}$, 537 G, 538-1), Tatu (CA 13, CA 34, MA 29) x Tatuí, Tatu (MA 21, CA 84) $x$ 'Tatu Amarelo', Tatu (CA 34) $x$ Roxo 47, Tatu (482) x Roxo 80-1, Tatu (CA 84) X (Tatuí X Arachis monticola), Tatu (CA 34) x (Tatuí $x$ A. villosa) e (Tatu 480) x Roxo 80-1) X (Tatuí x A. monticola).

Os experimentos foram delineados em blocos ao acaso com duas ou três repetições, sendo cada parcela constituída por uma linha de 5 ou $4 \mathrm{~m}$, espaçada de $0,60 \mathrm{~m}$. Na linha, a distância entre covas foi $0,10 \mathrm{~m}$, colocando-se 75 ou 60 sementes para deixar, após o desbaste, efetuado quinze dias após a emergência, $50 \mathrm{ou}$ 40 plantas por parcela de 5 ou $4 \mathrm{~m}$ respectivamente. Em todos os experimentos, usou-se como controle o 'Tatu'.
A adubação utilizada consistiu em $40 \mathrm{~kg}$ de $\mathrm{N}$ e $100 \mathrm{~kg}$ de $\mathrm{P}_{2} \mathrm{O}_{5}$ por hectare, na forma de sulfato de amônio aplicado em cobertura após o desbaste, e de superfosfato simples colocado no sulco antes da semeadura.

Para o controle de agentes patogênicos e pragas, foram efetuadas de duas a três pulverizações com uma mistura de Folidol ( $1 \mathrm{~cm}^{3} /$ litro), Endrin $\left(2 \mathrm{~cm}^{3} /\right.$ litro $)$ e Benlate $(0,7 \mathrm{~g} / 1 \mathrm{i}-$ tro), iniciadas após o desbaste e continuadas em intervalos de quinze a vinte dias.

No cultivo das águas de 1977 , instalaram-se quinze ensaios com 323 linhagens. Como a maioria dos experimentos foi perdida, principalmente pela má germinação das sementes em consequiência da falta de chuvas após a semeadura, estes ensaios foram novamente instalados em 1978.

Baseando-se nos dados de 1978 , apellas 107 linhagens tiveram suas produções novamente estimadas em 1979, em seis experimentos, sendo as demais descartadas. Desse modo, para as análises estatísticas e escolha daquelas que futuramente iräo constituir os ensaios regionais, apenas serão consideradas as produções das linhagens comuns em 1977, 1978 e 1979 ou 1978 e 1979 , após a verificação da homogeneidade dos quadrados médios residuais.

$O$ teste de Dunnett a $5 \%$ foi usado na comparação das médias das linhagens com a do controle 'Tatu'.

\section{RESULTADOS E DISCUSSĀO}

As produçōes e médias de vagens das linhagens derivadas do cruzamento de Tatuí x Roxo $80-1$, do 
Tatuí e do controle Tatu, no cultivo das águas de 1977, 1978 e 1979, acham-se no quadro 1 .
Observa-se que, no grupo de linhagens cuja produtividade média do Tatu foi $3.456 \mathrm{~kg} / \mathrm{ha}$, as linhagens

QUADRO 1 - Produçôes de frutos obtidas pelas linhagens de amendoim e pelo controle 'Tatu', nos ensaios das águas de 1977, 1978 e 1979, no Centro Experimental de Campinas

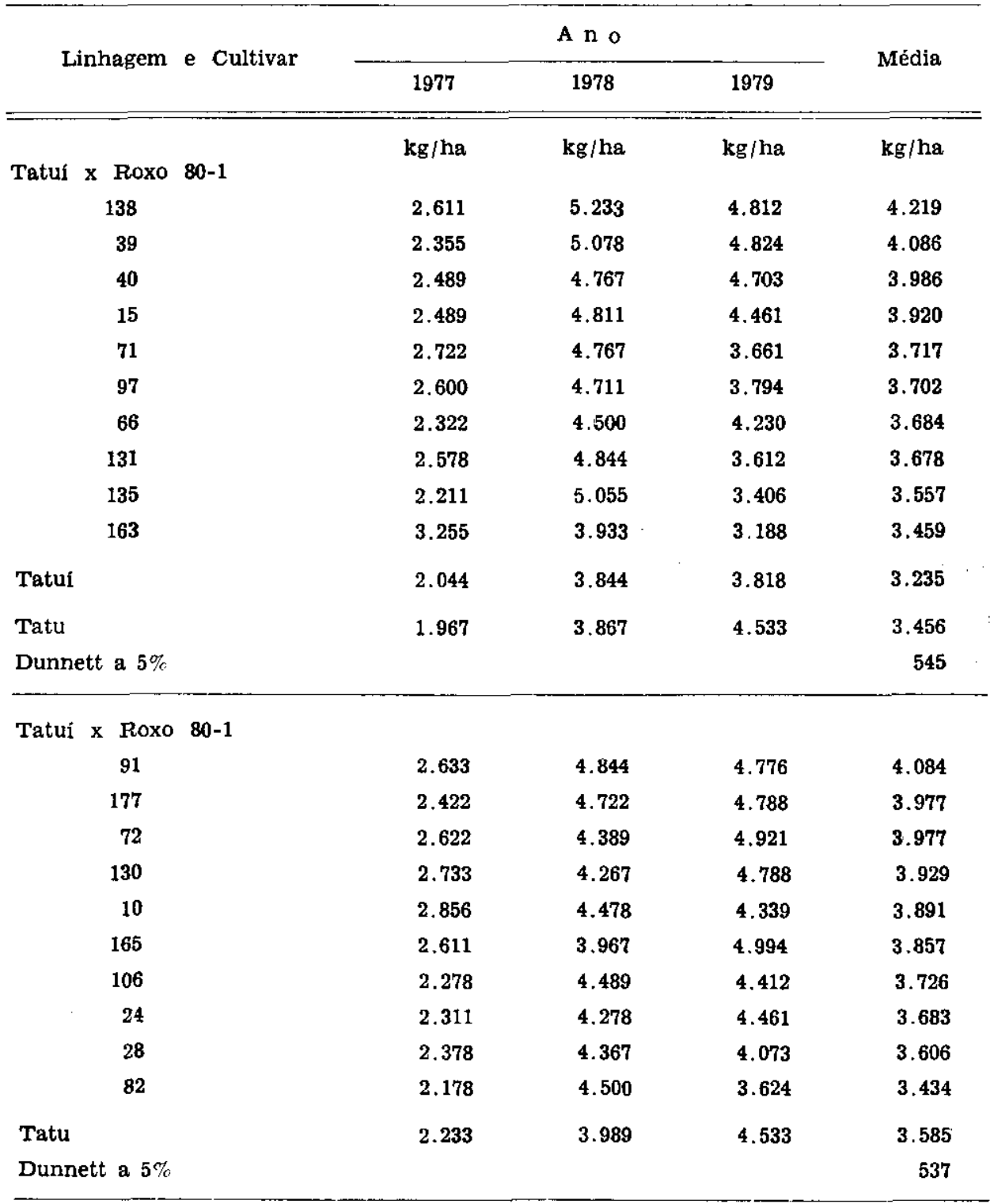


138 e 39 , estatisticamente superiores ao controle, destacaram-se das demais, com 4.219 e $4.086 \mathrm{~kg} / \mathrm{ha}$. A análise conjunta das produções mostrou efeitos significativos para tratamento, ano e interação tratamento $x$ ano. Isso mostra que, pelo menos algumas das linhagens diferem entre si quanto à produtividade e que as produçōes não foram uniformes no mínimo em um dos anos em estudo. As produções alcançadas pelas linhagens, pelo Tatuí e pelo Tatu em 1977, foram inferiores às de 1978 e 1979, devido à falta de chuvas durante boa parte do desenvolvimento das plantas, principalmente após a germinação. $O$ efeito significativo para a interação tratamento $x$ ano vem demonstrar que alguns dos tratamentos não exibiram o mesmo comportamento nos anos analisados.

Das linhagens que se encontram na parte inferior do quadro 1 , sobressaíram, por suas produções médias mais elevadas, as de números 91 , 177,72 e 130 , com $4.084,3.977$, 3.977 e $3.929 \mathrm{~kg} / \mathrm{ha}$. No entanto, essas produçóes não foram superiores, estatisticamente, à do controle Tatu, $3.585 \mathrm{~kg} / \mathrm{ha}$. O cfeito de ano foi significativo, o que não aconteceu para tratamento e para a interação tratamento $\mathrm{x}$ ano.

Do conjunto de linhagens em que o Tatu produziu, em média $3.518 \mathrm{~kg} /$ / ha, as linhagens 8,3 e 7 do cruzamento de Tatu (CA 34) x 538-1 e a 20, originária de Tatu (MA 29) $\mathrm{x}$ Tatuí com 4.553,4.439, 4.334 e $4.363 \mathrm{~kg} / \mathrm{ha}$ respectivamente, foram superiores ao controle (Quadro 2). Para essas linhagens, foram constatados os efeitos de tratamento e tratamento $\mathrm{x}$ ano.
No outro grupo de linhagens do quadro 2 , cuja produtividade média do Tatu foi $3.907 \mathrm{~kg} / \mathrm{ha}$, apenas a de número 32 , originária de Roxo $47 \mathrm{x}$ Tatu (CA 34), ultrapassou estatisticamente o controle: $4.730 \mathrm{~kg} / \mathrm{ha}$. A análise revelou efeitos significativos para ano e tratamento.

Produções médias de 5.366 e $5.315 \mathrm{~kg} / \mathrm{ha}$ das linhagens 26 (Tatu 482 x Roxo 80-1) e 102 (Tatuí $x$ Roxo 80-1), foram superiores à conseguida pelo Tatu: $4.318 \mathrm{~kg} / \mathrm{ha}$ (Quadro 2). Observaram-se efeitos significativos para tratamento, ano e tratamento $\mathrm{x}$ ano.

Verifica-se, ainda no quadro 2, que em geral as produçōes médias do Tatu, $3.518,3.907 \mathrm{e} 4.318 \mathrm{~kg} / \mathrm{ha}$, foram mais elevadas que as do Tatuí, $3.558,3.786$ e $3.802 \mathrm{~kg} / \mathrm{ha}$.

Do grupo de linhagens indicadas no quadro 3 , em que a produção média do Tatu foi $4.351 \mathrm{~kg} / \mathrm{ha}$, apenas a de número 86 , proveniente de $\mathrm{Ta}$ tuí x Roxo $80-1$, com $5.207 \mathrm{~kg} / \mathrm{ha}$, ultrapassou estatisticamente o controle.

Boas produções médias, 4.875 e $4.861 \mathrm{~kg} / \mathrm{ha}$, foram observadas para as linhagens 48 e 11 , resultantes de Tatuí x Roxo 80-1 e (Tatu-480 x Roxo-1) $x$ (Tatuí $x$ A. monticola). A análise das produções de 1978 e 1979 mostrou efeito significativo apenas para ano.

Produções médias de 5.067, 4.843 e $4.749 \mathrm{~kg} / \mathrm{ha}$ foram conseguidas pelas linhagens 182, $184 \mathrm{e}$ 201 do cruzamento de Tatuí x Roxo 80-1 (Quadro 3). No entanto, apenas as produções das linhagens 182 e 184 foram superiores à do Tatu, $3.949 \mathrm{~kg} /$ /ha. Mais uma vez, o efeito de 
QUADRO 2 - Produções conseguidas por linhagens de amendoim 'Tatui' e pelo controle 'Tatu', nos ensaios das águas de 1978 e 1979, em Campinas

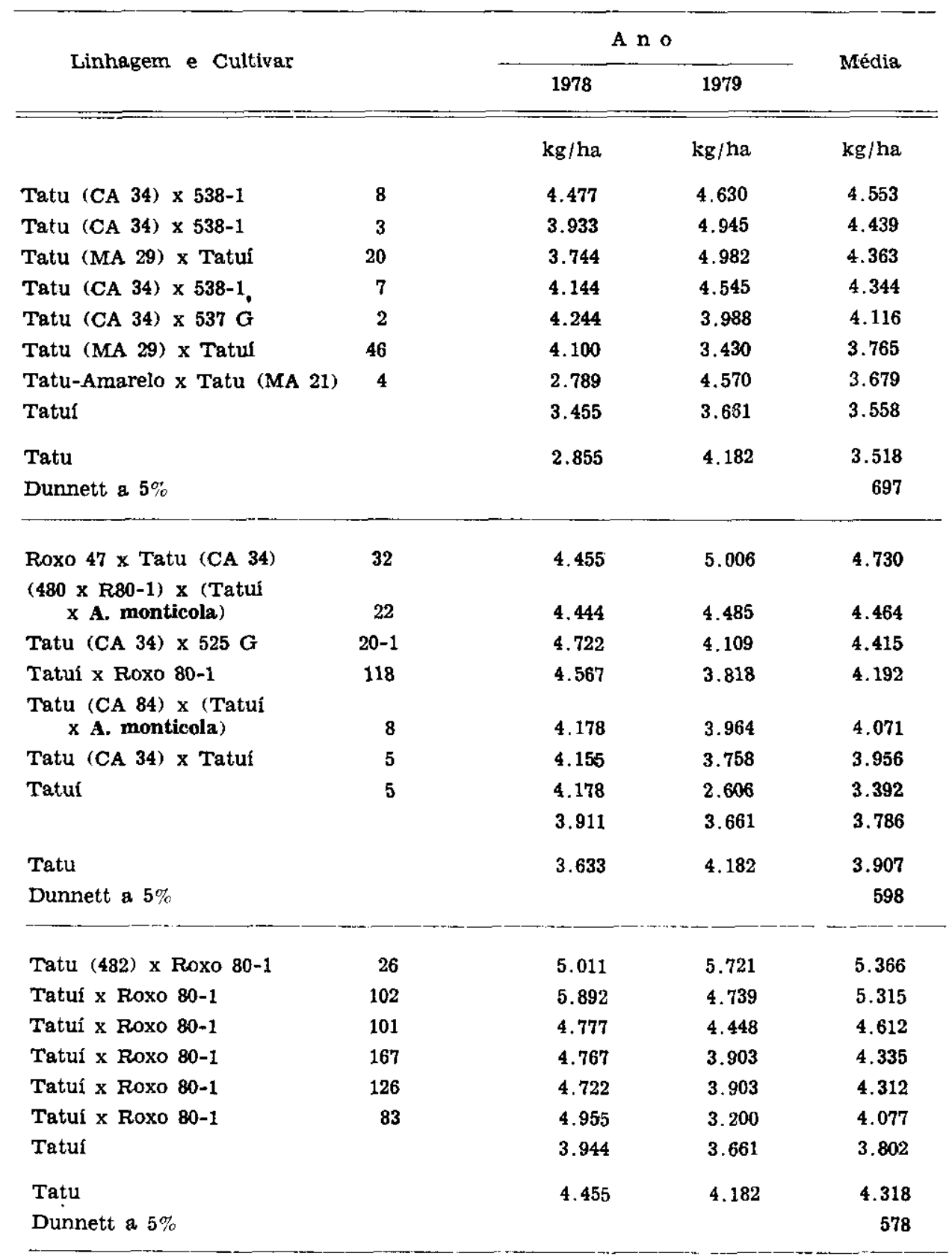


QUADRO 3 - Produçōes de frutos das linhagens de amendolm, do 'Tatuí' e do controle 'Tatu', no plantio das águas de 1978 e 1979, no Centro Experimental de Campinas

\begin{tabular}{|c|c|c|c|c|}
\hline \multirow{2}{*}{ Linhagem e Cultivar } & & \multicolumn{2}{|c|}{ A n o } & \multirow{2}{*}{ Média } \\
\hline & & 1978 & 1979 & \\
\hline & & $\mathrm{kg} / \mathrm{ha}$ & $\mathrm{kg} / \mathrm{ha}$ & $\mathrm{kg} / \mathrm{ha}$ \\
\hline Tatuí x Roxo 80-1 & 86 & 5.578 & 4.836 & 5.207 \\
\hline Tatuí $\mathrm{x}$ Roxo 80-1 & 48 & 4.744 & 5.006 & 4.875 \\
\hline $\begin{array}{l}\text { (Tatu } 480 \times \text { Roxo 80-1) } x \\
\text { (Tatuí } \times \text { A. monticola) }\end{array}$ & 11 & 5.055 & 4.667 & 4.861 \\
\hline Tatu (CA 13) x Tatú & 29 & 4.611 & 4.909 & 4.760 \\
\hline Tatu (CA 13) x Tatuí & 15 & 4.800 & 4.570 & 4.685 \\
\hline Tatuí x Roxo 80-1 & 45 & 4.755 & 4.448 & 4.601 \\
\hline Tatuí x Roxo 80-1 & $131-1$ & 4.955 & 4.024 & 4.489 \\
\hline Tatu (CA 34) $\times 525 \mathrm{G}$ & $20-1$ & 4.844 & 4.121 & 4.482 \\
\hline Tatuí x Roxo 80-1 & 3 & 4.789 & 4.024 & 4.406 \\
\hline Tatui & & 3.689 & 4.121 & 3.905 \\
\hline Tatu & & 4.400 & 4.303 & 4.351 \\
\hline Dunnett a $\mathbf{5} \%$ & & & & 741 \\
\hline
\end{tabular}

Tatui $\mathrm{x}$ Roxo 80-1

$\begin{array}{llll}182 & 5.104 & 5.030 & 5.067 \\ 184 & 5.354 & 4.333 & 4.848 \\ 201 & 4.937 & 4.561 & 4.749 \\ 264 & 4.799 & 4.015 & 4.407 \\ 250 & 5.104 & 3.667 & 4.385 \\ 256 & 4.792 & 3.818 & 4.305 \\ 253 & 5.042 & 3.485 & 4.263 \\ 227 & 4.583 & 3.682 & 4.132 \\ 249 & 4.958 & 3.333 & 4.145 \\ & & & 4.371 \\ & 4.895 & 3.848 & \\ & & & 3.949\end{array}$

Dunnett a $5 \%$ 
ano foi significativo, o que não ocorreu com tratamento e com a interaçăo tratamento $x$ ano.

As produções médias do Tatu, 4.351 e $3.949 \mathrm{~kg} / \mathrm{ha}$, praticamente se equivaleram às do Tatuí, $3.905 \mathrm{e}$ $4.371 \mathrm{~kg} / \mathrm{ha}$ (Quadro 3).

Produções de 4.756 e $4.658 \mathrm{~kg} /$ /ha das linhagens 1 e 25 de Tatu (CA 84) $x$ Tatu-Amarelo e Tatu (CA 34) x 538-1, foram as mais elevadas para o grupo no qual o Tatu produziu, em média, $4.127 \mathrm{~kg} / \mathrm{ha}$ (Quadro 4). As produções das linhagens se equivalem, estatisticamente, à do controle.

Produções médias superiores à do Tatu, $4.401 \mathrm{~kg} / \mathrm{ha}$, foram obtidas pelas linhagens $5,31,6$ e 32 (Quadro 4), originárias do cruzamento de Tatu (CA 84) x Tatu-Amarelo, com $5.684,5.629,5.376$ e $5.332 \mathrm{~kg} / \mathrm{ha}$.

Para os dois conjuntos de linhagens do quadro 4 , a análise estatística mostrou apenas efeito significativo para ano.

Excelentes produções médias foram conseguidas pelas linhagens 238 , 34 e 261 (Quadro 5), 6.445, 5.491 e $5.486 \mathrm{~kg} / \mathrm{ha}$, embora somente a primeira tenha sido superior, estatisticamente, à do Tatu, $4.887 \mathrm{~kg} / \mathrm{ha}$.

Para o outro grupo de linhagens (Quadro 5), em que o Tatu produziu, em média, $4.612 \mathrm{~kg} / \mathrm{ha}$, a de número 17, do cruzamento Tatu (CA 84) $x$ Tatu-Amarelo, e a 41, de Roxo $47 \mathrm{x}$ Tatu (CA 34), com 5.666 e $5.400 \mathrm{~kg} /$ /ha, foram superiores ao controle. Destacou-se, também, a linhagem 26 (MA $29 \times$ Tatuí), com produção média de $5.212 \mathrm{~kg} / \mathrm{ha}$.

As análises estatísticas revelaram, para esses dois grupos de linha- gens, efeitos significativos para tratamento e para ano.

Produções médias elevadas (Quadro 6) foram obtidas pelas linhagens 205 e 196, de Tatuí x R80-1, 5.539 e $5.350 \mathrm{~kg} / \mathrm{ha}$. Destas, somente a primeira foi superior à do Tatu, que obteve $4.764 \mathrm{~kg} / \mathrm{ha}$. Efeitos significativos foram notados para tratamento e para ano.

Das linhagens do quadro 6 , cujo controle, Tatu, obteve em média $4.459 \mathrm{~kg} / \mathrm{ha}$, destacaram-se os de números 34 e 2 , resultantes dos cruzamentos Tatu (CA 84) x Tatu-Amarelo e Tatu (CA 34) x (Tatuí x Arachis villosa), com 6.062 e $5.288 \mathrm{~kg} /$ ha respectivamente. Ambas foram superiores ao Tatu em produtividade. A análise estatística conjunta para 1978 e 1979 mostrou efeito significativo apenas para tratamento.

Nos experimentos do quadro 6 , as produções médias do Tatu (4.764 e $4.459 \mathrm{~kg} / \mathrm{ha}$ ) foram mais elevadas que as do Tatuí $(3.806 \mathrm{e}$ $3.971 \mathrm{~kg} / \mathrm{ha}$ ).

Os resultados mostraram que 22 linhagens com produções médias de 4.219 a $6.445 \mathrm{~kg} / \mathrm{ha}$ foram superiores, estatisticamente, às observadas para o cultivar Tatu, que variaram de 3.456 a $4.887 \mathrm{~kg} / \mathrm{ha}$. Algumas dessas linhagens, como, por exemplo, 86, 138 e 238 , originárias de Tatuí $x$ Roxo $80-1$, e 5, 17, 34, de Tatu x TatuAmarelo, apresentam, na sua quase totalidade, frutos com duas sementes com tegumento vermelho. Outras, como 102 e 182 , do cruzamento de Tatuí x Roxo 80-1, mostram a mesma característica quanto aos frutos, porém com sementes de película creme, maiores do que as do Tatu e do Tatuí. 
QUADRO 4 - Produçōes de frutos conseguidas pelas linhagens de amendoim $e$ pelo controle 'Tatu', nos experimentos instalados nas águas de 1978 e 1979, em Campinas

Linhagem e Cultivar $\begin{gathered}\text { A n o } \\ 1978 \\ \cdots\end{gathered}$

\begin{tabular}{|c|c|c|c|c|}
\hline & & $\mathrm{kg} / \mathrm{ha}$ & $\mathrm{kg} / \mathrm{ha}$ & $\mathrm{kg} / \mathrm{ha}$ \\
\hline Tatu (CA 84) x Tatu-Amarelo & 1 & 5.042 & 4.470 & 4.756 \\
\hline Tatu (CA 34) x 538-1 & 25 & 4.937 & 4.379 & 4.658 \\
\hline Tatu (CA 84) x Tatu-Amarelo & 11 & 4.875 & 4.151 & 4.513 \\
\hline Tatu (MA 29) x Tatuí & 28 & 5.125 & 3.848 & 4.486 \\
\hline Tatu (MA 29) x Tatuí & 24 & 5.000 & 3.757 & 4.378 \\
\hline Tatu (CA 84) x Tatu-Amarelo & 16 & 4.812 & 3.697 & 4.254 \\
\hline Tatu (CA 84) x Tatu-Amarelo & 19 & 4.270 & 3.227 & 3.748 \\
\hline Tatu & & 4.042 & 4.212 & 4.127 \\
\hline Dunnett a $\mathbf{5 \%}$ & & & & 794 \\
\hline Tatu (CA 84) x Tatu-Amarelo & 5 & 6.500 & 4.869 & 5.684 \\
\hline Tatu (CA 84) x Tatu-Amarelo & 31 & 5.278 & 5.980 & 5.629 \\
\hline Tatu (CA 84) x Tatu-Amarelo & 6 & 5.722 & 5.030 & 5.376 \\
\hline Tatu (CA 84) x Tatu-Amarelo & 32 & 5.472 & 5.192 & 5.332 \\
\hline Tatu (CA 84) x Tatu-Amarelo & 7 & 5.528 & 5.030 & 5.279 \\
\hline Tatu (CA 84) $\times$ Tatu-Amarelo & 10 & 5.333 & 5.131 & 5.232 \\
\hline Tatu (CA 34) $\times 357 \mathrm{G}$ & 10 & 5.389 & 4.990 & 5.189 \\
\hline Tatu (CA 34) x 357 G & 26 & 5.557 & 4.586 & 5.071 \\
\hline Tatu (CA 34) x 538-1 & 17 & 5.389 & 4.667 & 5.028 \\
\hline Tatu (CA 84) $\times$ Tatu-Amarelo & 15 & 5.472 & 4.586 & 5.029 \\
\hline Tatu (CA 84) x Tatu-Amarelo & 16 & 5.333 & 4.667 & 5.000 \\
\hline Tatu (CA 84) x Tatu-Amarelo & 21 & 5.361 & 4.323 & 4.842 \\
\hline Tatu & & 4.500 & 4.303 & 4.401 \\
\hline Dunnett a $\mathbf{5} \%$ & & & & 914 \\
\hline
\end{tabular}


QUADRO 5 - Capacidade produtiva de vagens de linhagens de amendoim e do controle 'Tatu', nos ensaios plantados nas águas de 1978 e 1979, no Centro Experimental de Campinas

Linhagem e Cultivar

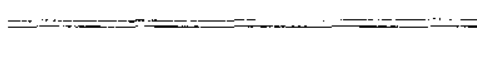

Tatui $x$ Roxo 80-1

238

34

261

257

232

36

190

244

258

Tatu

Dunnett a $5 \%$

$482 \times$ Roxo 80-1

Tatuí x Roxo 80-1
A n o

1978

1979 $\mathrm{kg} / \mathrm{ha}$

$\mathrm{kg} / \mathrm{ha}$

5.697

6.445

7.194

5.454

5.491

6

5.528

$4.667^{--}$

5.486

6.306

4.909

5.385

5.862

4.747

5.304

5.862

4.687

5.274

5.862

4.768

5.037

5.306

3.879

4.786

5.694

3.273

4.442

5.611

4.303

4.887

5.472

4.303

807

\begin{tabular}{lllll} 
Tatu (CA 84) x Tatu-Amarelo & 17 & 6.222 & 5.111 & 5.666 \\
Roxo 47 x Tatu (CA 34) & 41 & 5.528 & 5.273 & 5.400 \\
Tatu (MA 29) x Tatui & 26 & 6.222 & 4.202 & 5.212 \\
Tatu (CA 34) x Tatui & 18 & 5.389 & 4.727 & 5.058 \\
Tatu (MA 21) x Tatu-Amarelo & 21 & 5.583 & 4.323 & 4.953 \\
Tatu (MA 29) x Tatuf & 25 & 4.889 & 3.434 & 4.161 \\
Tatu & & 4.639 & 4.586 & 4.612 \\
Dunnett a 5\% & & & & 615 \\
\hline
\end{tabular}


Vol. 41 , Art. n. ${ }^{\circ} 8$

QUADRO 6 - Produçōes de vagens obtidas pelas linhagens de amendoim, por 'Tatui' e pelo controle 'Tatu', nos ensaios instalados no cultivo das águas de 1978 e 1979, em Campinas

\section{Linhagem e Cultivar}

A $\mathrm{n} O$

$1978 \quad$ Média

Tatui x Roxo 80-1

$\begin{array}{rrrr}205 & 6.028 & 5.050 & 5.539 \\ 196 & 5.833 & 4.868 & 5.350 \\ 178 & 6.222 & 4.323 & 5.272 \\ 199 & 5.972 & 4.565 & 5.268 \\ 188 & 5.833 & 4.484 & 5.158 \\ 212 & 5.750 & 4.565 & 5.157 \\ 193 & 5.555 & 3.919 & 4.737 \\ 204 & 6.139 & 3.111 & 4.625 \\ & 4.139 & 3.474 & 3.806 \\ & & & 4.764 \\ & 4.944 & 4.585 & 767\end{array}$

Dunnett a $5 \%$

767

Tatu (CA 84) x Tatu-Amarelo

7.033

5.091

6.062

Tatu (CA 34) $\times$ (Tatú $x$
A. villosa)
2
5.000
5.576
5.288

14

5.267

4.687

4.977

Tatu (CA 84) $\times$ Tatu-Amarelo 18

5.233

4.626

4.929

Tatu (CA 84) x Tatu-Amarelo

5.033

4.768

4.900

$482 \times$ Roxo $80-1$

40

5.967

3.757

4.862

$482 \times$ Roxo 80-1

47

4.900

4.505

4.702

Tatuí

4.467

3.475

3.971

Tatu

4.333

4.586

4.459

Dunnett a $\mathbf{5 \%}$ 
As produções médias obtidas por essas linhagens demonstram o seu potencial para o aumento da produtividade dessa cultura, levando-se em consideração que o rendimento médio observado no Estado de São Paulo foi $1.319 \mathrm{~kg} / \mathrm{ha}$ em 1978 (2). Conjuntamente a essa produtividade, aliam-se as características de fruto e semente que as tornam mais atrativas aos agricultores, em relação ao cultivar Tatu.

O efeito de ano, observado na majoria das análises, pode ser explicado em conseqüência da estiagem observada em 1977, das excelentes condições para o desenvolvimento das plantas e produção de 1978, e pelo excesso de chuvas em 1979, que dificultou o controle fitossanitário, fazendo com que as produções fossem menores do que as obtidas em 1978.

Em relação ao efeito da interação tratamento $\mathrm{x}$ ano, o qual foi significativo em apenas três das treze análises conjuntas efetuadas, pode-se dizer que talvez o comportamento diferencial das linhagens em 1978 e 1979 se deva a uma diferença de nível de resistência, principalmente aos fungos Cercospora arachidicola e Cercosporidium personatum, causadores das manchas castanha e preta no amendoim. No entanto, é bastante difícil diferenciar, visualmente, níveis de resistência a esses patógenos em condições de campo, a não ser quando as diferenças são bastante acentuadas entre as linhagens.

No período 1977-1979, a produtividade média do Tatu, $4.090 \mathrm{~kg} / \mathrm{ha}$, foi mais elevada que a do Tatuí, $3.804 \mathrm{~kg} / \mathrm{ha}$.

As melhores linhagens serão incluídas nos ensaios regionais a serem instalados nas principais regiōes produtoras de amendoim no Estado de São Paulo, enquanto sementes de algumas delas, as quais não mostraram tendência de interação como ano, serão aumentadas em quantidade e colocadas à disposição dos agricultores.

\section{YIELDING CAPACITY OF NEW PEANUT LINES (ARACHIS HXPOGAEA)}

\section{SUMMARY}

Aiming at the knowledge of the yielding capacity of new peanut lines, 36 trials in randomized complete-block design, using 'Tatu' as control were carried out in the Main Experimental Station of Campinas, during the rainy season of 1977, 1978 and 1979.

The peanut lines originated from individual selection in the crosses of Tatuí $x$ Roxo 80-1, Tatu $x$ Introduction, Tatu $x$ Tatuí, Tatu $x$ Tatu Amarelo, Tatu $x$ Roxo 47, Tatu $\times$ Roxo 80-1, Tatu $x$ (Tatuf $x$ Arachis monticola), Tatu $x$ (Tatuí $x$ A. villosa) and (Tatu $x$ Roxo 80-1) X (Tatuí $x$ A. monticola).

The statistical analyses showed that 22 lines with means of 4,219 to $6,445 \mathrm{~kg} / \mathrm{ha}$ were superior to those observed for Tatu, which ranged from 3,456 to $4,887 \mathrm{~kg} / \mathrm{ha}$. In thirteen analyses the effects of year and treatment $x$ year were significant in eleven and three of them, respectively.

The best lines will be included in the regional trials and seeds of some of them increased in large quantities for releasing to the growers. 


\section{REFERENCIAS BIBLIOGRAFICAS}

1. FOOD AGRICULTURE ORGANIZATION. Production Yearbook. Roma, FAO, 1978. v.32, p.127.

2. INSTITUTO BRASILEIRO DE GEOGRAFIA E ESTATISTICA. Anuário Estatístico do Brasil. Rlo de Janeiro, IBGE, 1978. v.39, p.362 e 375. 